\title{
Optimization of reproductive management programs using lift chart analysis and cost-sensitive evaluation of classification errors
}

\author{
Saleh Shahinfar, ${ }^{* 1}$ Jerry N. Guenther, ${ }^{*}$ C. David Page,† Afshin S. Kalantari,, Victor E. Cabrera, \\ Paul M. Fricke, ${ }^{*}$ and Kent A. Weigel* \\ *Department of Dairy Science, and \\ †Department of Biostatistics and Medical Informatics and Department of Computer Science, University of Wisconsin, Madison 53706
}

\begin{abstract}
The common practice on most commercial dairy farms is to inseminate all cows that are eligible for breeding, while ignoring (or absorbing) the costs associated with semen and labor directed toward low-fertility cows that are unlikely to conceive. Modern analytical methods, such as machine learning algorithms, can be applied to cow-specific explanatory variables for the purpose of computing probabilities of success or failure associated with upcoming insemination events. Lift chart analysis can identify subsets of high fertility cows that are likely to conceive and are therefore appropriate targets for insemination (e.g., with conventional artificial insemination semen or expensive sex-enhanced semen), as well as subsets of low-fertility cows that are unlikely to conceive and should therefore be passed over at that point in time. Although such a strategy might be economically viable, the management, environmental, and financial conditions on one farm might differ widely from conditions on the next, and hence the reproductive management recommendations derived from such a tool may be suboptimal for specific farms. When coupled with cost-sensitive evaluation of misclassified and correctly classified insemination events, the strategy can be a potentially powerful tool for optimizing the reproductive management of individual farms. In the present study, lift chart analysis and cost-sensitive evaluation were applied to a data set consisting of 54,806 insemination events of primiparous Holstein cows on 26 Wisconsin farms, as well as a data set with 17,197 insemination events of primiparous Holstein cows on 3 Wisconsin farms, where the latter had more detailed information regarding health events of individual cows. In the first data set, the gains in profit associated with limiting inseminations to subsets of 79
\end{abstract}

Received April 19, 2014

Accepted February 23, 2015.

${ }^{1}$ Corresponding author: shahinfar@wisc.edu to $97 \%$ of the most fertile eligible cows ranged from $\$ 0.44$ to $\$ 2.18$ per eligible cow in a monthly breeding period, depending on days in milk at breeding and milk yield relative to contemporaries. In the second data set, the strategy of inseminating only a subset consisting of $59 \%$ of the most fertile cows conferred a gain in profit of $\$ 5.21$ per eligible cow in a monthly breeding period. These results suggest that, when used with a powerful classification algorithm, lift chart analysis and cost-sensitive evaluation of correctly classified and misclassified insemination events can enhance the performance and profitability of reproductive management programs on commercial dairy farms.

Key words: machine learning, reproductive management, lift chart analysis, cost-sensitive evaluation, dairy cattle

\section{INTRODUCTION}

The underlying physiological factors that determine the success or failure of an insemination event in dairy cows are complex and often unknown. Conception rate has a major effect on farm profitability because it affects the number of replacement heifers needed to maintain herd size, the capacity to generate and sell extra heifers, the proportion of pregnant cows in the herd, average milk production of these cows, insemination and veterinary costs, involuntary culling rate, and other factors (Britt, 1985; Meadows et al., 2005; Giordano et al., 2011).

Previous studies have attempted to predict the outcome of an insemination event in lactating dairy cows based on results of ovarian palpation (Ludwick and Rader, 1967), interactions between stage of lactation and pregnancy status (Sharma et al., 1990), or levels of NEFA and blood glucose (Garverick et al., 2013). Other studies have considered reproductive management programs from an economic point of view; for example, by estimating the economic value of a pregnancy (De Vries, 2006), finding an economically optimal voluntary waiting period (Inchaisri et al., 2011), or carrying out 
an economic comparison of natural service and timed AI programs (Lima et al., 2010; Giordano et al., 2012).

On most commercial dairy farms, it is common practice to inseminate all cows that are eligible for breeding, with the hope that modest conception rates coupled with high service rates will lead to efficient overall reproductive performance. This strategy may be economically sensible when insemination costs, specifically semen and technician service, are low. However, evidence that this strategy maximizes farm profitability is lacking, and insemination costs can be relatively high if sex-enhanced semen is used. Several studies have indicated that targeted reproductive management of specific (groups of) cows could be more profitable (e.g., Giordano et al., 2012), but no study to date has attempted to predict the outcomes of insemination events for individual cows before the breeding while subsequently evaluating the benefits and costs of correct and incorrect breeding decisions, respectively.

Shahinfar et al. (2014b) evaluated the classification accuracy of several machine learning algorithms when predicting the outcome of an insemination event for an individual cow based on the production, reproduction, health, and genetic information available before the insemination. The proportions of insemination events that were classified correctly as successes or failures were $72.3 \%$ for primiparous cows and $73.6 \%$ for multiparous cows in 5-fold cross-validation using a random forest algorithm. Classification accuracies of this magnitude bring into question the practice of inseminating every eligible cow, while ignoring the vast amount of information available about each cow's genetic potential, health history, and lactation performance. Furthermore, we can carry out a lift chart analysis (Witten and Frank, 2005) to identify a subset of cows with much greater probability of conception than the entire pool of eligible cows. Although such an analysis would, for example, facilitate the use of expensive sex-enhanced or high-genetic-merit semen for mating the subset of cows most likely to conceive, it would be limited by the fact that evaluation of algorithms based only on classification accuracy implies that the benefits and costs associated with all correctly classified or misclassified events are equal. In reality, the costs and benefits may differ considerably. For example, if insemination costs are low, the cost of failing to inseminate a cow that would have conceived (i.e., a false negative, $\mathbf{F N}$ ) is likely to be greater than the cost of inseminating a cow that will not conceive (i.e., a false positive, FP). Moreover, the benefit derived from inseminating a cow that will conceive (i.e., a true positive, TP) may be greater than the benefit associated with failing to inseminate a cow that would not have conceived (i.e., a true negative, $\mathbf{T N}$ ).
The objective of this study was to build upon the work of Shahinfar et al. (2014b), which showed that relatively high accuracy of classifying insemination events as successes or failures could be achieved before insemination. Lift chart analysis was used to identify subsets of cows with high probability of conception, and then cost-sensitive evaluation was used to assess the economic benefits that might be achieved. Implementing such an approach for reproductive management of lactating dairy cows on commercial farms can lead to the development of an on-farm decision support tool that can be used by reproductive management consultants or incorporated into herd management software programs.

\section{MATERIALS AND METHODS}

\section{Data}

Two data sets were used in the analyses presented herein. The first, which will be denoted as Data_1, consisted of 54,806 insemination events from 22,210 primiparous Holstein cows that were inseminated between 2000 and 2010 in 26 Alta Genetics (Watertown, WI) Advantage Program herds located throughout Wisconsin. These breeding records represented a subset of the data used by Shahinfar et al. (2014b), in which insemination events corresponded to 3 intervals of DIM: $60-90,90-120$, or $120-150$, and 3 ranges of relative milk yield (RMY): between 18 and $6 \%$ below the within-herd mean, between $6 \%$ below and $6 \%$ above the within-herd mean, or between 6 and $18 \%$ above the within-herd mean. Mean conception rate in Data_1 was $32 \%$, and individual herds ranged from 18 to $44 \%$. Additional details about genetic predisposition, health history, and lactation performance of herds in Data_1 are provided in Shahinfar et al. (2014b).

The second, which will be denoted as Data_2, consisted of 17,197 insemination events from 5,356 primiparous Holstein cows that were inseminated between 2002 and 2013 in 3 additional herds also located in Wisconsin. These herds were chosen because they had more complete data regarding common early postpartum health disorders (i.e., mastitis, ketosis, lameness, displaced abomasum, metritis, and retained placenta) than the herds in Data_1. In contrast to Data_1, because of the small size of the data set and the need for a more general model, insemination events in Data_2 were not stratified by DIM or RMY, and all insemination events were analyzed jointly. The range in DIM at time of insemination was 12 to 479 in Data_2. Mean across-herd conception rate in Data_2 was $42 \%$, and means for individual herds ranged from 38 to $45 \%$. 
As in Shahinfar et al. (2014b), 25 potential explanatory variables related to genetic predisposition, health history, and lactation performance that were known for each cow before insemination were considered when classifying insemination outcomes in Data_1 and Data_2. These included mean conception rate within the herd during the previous $3 \mathrm{mo}$, age at calving, stage of lactation, DIM at first breeding, number of times bred during the current lactation, number of times bred as a yearling heifer, breeding protocol utilized, sire's PTA for daughter pregnancy rate, service sire's PTA for daughter pregnancy rate, service sire's PTA for sire conception rate, inbreeding coefficient of the cow, sex of previous calf, fat:protein ratio at last test, ECM at last test, SCS at last test, and incidences of dystocia, mastitis, ketosis, retained placenta, lameness, displaced abomasum, and twinning in the current lactation. In the present study, herd, year, and month of insemination were considered as separate explanatory variables, whereas the study of Shahinfar et al. (2014b) utilized herd-year-month as an explanatory variable.

\section{Classification}

Machine learning is a branch of artificial intelligence in which computer algorithms learn from the data without prior assumptions about statistical models or distributions. These algorithms discover patterns in the training data and form generalized rules for prediction, classification, or clustering of data that have not yet been observed. As opposed to prediction of a continuous variable (e.g., by regression on a set of explanatory variables), classification involves partitioning the state space into 2 or more discrete and mutually exclusive categories (Mitchell, 1997). For example, decision tree uses a divide-and-conquer method based on gain ratio to classify instances into categories, such as classifying future insemination events into pregnant and nonpregnant outcomes (Shahinfar et al., 2014b).

\section{Probability of Conception}

Because of its superior classification accuracy relative to other machine learning algorithms considered in Shahinfar et al. (2014b), a random forest algorithm was used in the present study. Because this is an ensemble algorithm in which numerous classifiers are trained by repeatedly sampling from the original data, numerous decision trees are generated. Therefore, the probability of conception for a particular insemination event corresponding to a given cow represents the aggregated probability across all trees and forests generated by the algorithm. In the present study, 10-fold cross-validation was carried out within each of the 9 subclasses in
Data_1 that corresponded to a given range in DIM and a given level of RMY, and all 54,806 insemination events were included in the analysis. Within each subclass, the proportion of correctly classified insemination events was calculated within each fold, and these were subsequently averaged across folds to determine classification accuracy. In addition, the area under the receiver operating characteristic (ROC) curve, which has $\mathrm{FP}$ rate on the $\mathrm{x}$-axis and $\mathrm{TP}$ rate on the $\mathrm{y}$-axis, was computed for each of the 9 subclasses.

In contrast, Data_2 was randomly subdivided into 2 partitions. The first contained 14,000 insemination events, and this partition of the data was used to train the model and learn its parameters using 10-fold cross-validation, as described previously for Data_1. The second contained 3,197 randomly chosen insemination events, and the purpose of creating this partition was to mimic a real-life situation in which a decisionmaking tool was initially developed and tuned using an established database (e.g., at a breeding company) and subsequently applied to new data on a commercial farm (e.g., by a field technician). Therefore, the outcomes of the 3,197 insemination events in the second partition were masked throughout the training process; at the time of insemination, data regarding the corresponding explanatory variables were fed into the model developed from the 14,000 events in the first partition to compute the probability of success or failure for each insemination event.

All analyses were carried out on a monthly basis because variables such as fat:protein ratio and ECM are typically measured monthly in DHI milk recording programs. Therefore, we assumed, for instance, that a cow inseminated in one month could be declared as pregnant or re-inseminated in the following month, and a cow that was not inseminated in the current month could be inseminated or passed over a second time in the following month. All explanatory variables regarding the genetic potential, health history, and lactation performance of a given cow were updated monthly, such that a cow with a low probability of conception in a given month would tend to have low probability of conception in the subsequent month, but not strictly so. Likewise, a cow with high probably of conception in one month would tend to have a high probability of conception in the following month, but this probability could decrease due to lameness, high SCS, or other factors. In practice, one could consider a longer or shorter interval (e.g., 21 d) for compiling data regarding explanatory variables and generating predictions of insemination outcomes. In herds with daily milk recording and automated measurement of physical activity and rumination patterns, it would be possible to carry out such an analysis daily. 


\section{Lift Chart Analysis}

In commercial applications of data-mining algorithms that involve the marketing of consumer products, a lift chart analysis is often used to predict the increase in response rate that would be achieved by targeting a specific demographic group, as opposed to blanket marketing of a given product to the entire population of consumers (Shin and Cho, 2006; Chen et al., 2011; Sheng et al., 2014). In the context of reproductive management of dairy herds, a lift chart analysis can be used to predict the gain in conception rate that would be achieved by inseminating a selected subset of cows with high probability of conception, as opposed to inseminating all eligible cows in the herd. For example, consider a herd with 1,000 cows that are eligible for insemination and assume that the mean conception rate for the entire herd is 0.30 . If we inseminate all of the eligible cows, $70 \%$ will not conceive, and our 1,000 inseminations will yield 300 pregnancies. However, if we have a classification algorithm that is capable of identifying a subset of 400 cows for which the expected conception rate will be 0.60 , we can limit our inseminations to this highly fertile subset and, by doing so, we can generate 240 pregnancies from only 400 inseminations.
In practical applications of such an analysis, we would have a classification algorithm that would predict the probability of success or failure for each potential insemination event, and we could rank these events according to their respective probabilities of success. Therefore, we could consider a wide range of scenarios; for example, we may be faced with the decision of whether to inseminate a subset of 400 cows with an expected conception rate of $60 \%$ or a subset of 700 cows with an expected conception rate of $45 \%$. This concept is illustrated in the lift chart shown in Figure 1 , in which the proportion of cows to be inseminated is shown on the $\mathrm{x}$-axis, and the number of anticipated pregnancies is shown on the y-axis. The gain in conception rate within a given subset, relative to the conception rate of the entire population of eligible cows, is known as the lift factor, which for the examples shown above would be $(60 \% \div 30 \%)=2.0$ and $(45 \% \div 30 \%)$ $=1.5$, respectively.

\section{Cost-Sensitive Evaluation}

Optimizing classification accuracy in a lift chart analysis, as described above, can be misleading if one does not consider the benefits and costs associated with

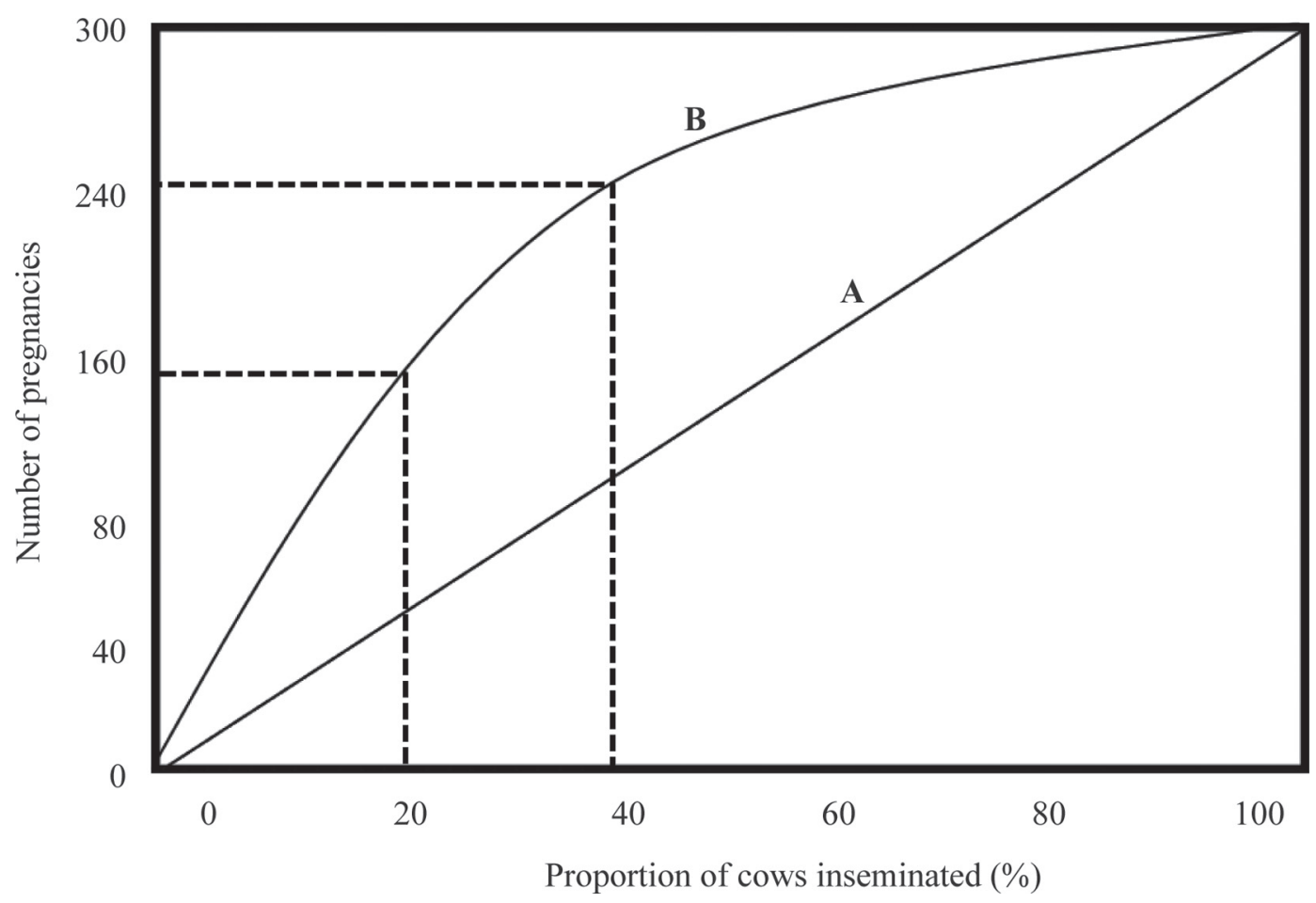

Figure 1. Conceptual illustration of a lift chart analysis for the classification problem of predicting the success or failure of an insemination event, as carried out in the present study. Line A shows the number of pregnancies that would be generated by inseminating a random subset of cows of a given size, whereas curve B shows the number of pregnancies that would be generated by inseminating subsets of cows of varying size (e.g., 20 or 40\%) after ranking the insemination events from highest to lowest based on probability of conception. 
Table 1. Conceptual illustration of cost-benefit matrix for classifying upcoming insemination events as successes or failures based on a set of explanatory variables ${ }^{1}$

\begin{tabular}{|c|c|c|}
\hline \multirow[b]{2}{*}{ Actual outcome } & \multicolumn{2}{|r|}{ Predicted classification } \\
\hline & Pregnant & Nonpregnant \\
\hline Pregnant & $\begin{array}{l}\mathrm{TP} \\
\text { (pregnancy value) }\end{array}$ & $\begin{array}{l}\text { FN } \\
\text { (difference between } 2 \text { subsequent pregnancy values) }\end{array}$ \\
\hline Nonpregnant & $\begin{array}{l}\text { FP } \\
\text { (insemination cost) }\end{array}$ & $\begin{array}{l}\mathrm{TN} \\
(0)\end{array}$ \\
\hline $\begin{array}{l}{ }^{1} \text { The benefits an } \\
\text { rentheses, includ } \\
\text { became pregnant } \\
\text { become pregnant } \\
\text { become pregnant } \\
\text { become pregnant }\end{array}$ & $\begin{array}{l}\text { assigned to each ty } \\
\text { nefits assigned to tr } \\
\text { e negative (TN) cla } \\
\text { minated; a false pos } \\
\text { false negative (FN } \\
\text { minated. }\end{array}$ & $\begin{array}{l}\text { classification decision in the present study are shown } \\
\text { itive (TP) classification of a cow that was inseminat } \\
\text { ion of a cow that was not inseminated and would no } \\
\text { FP) classification of a cow that was inseminated but } \\
\text { ification of a cow that was not inseminated but woul }\end{array}$ \\
\hline
\end{tabular}

the various types of correctly classified or misclassified outcomes, specifically TP, TN, FP, and FN. It is naïve to assume that the costs of FP and FN errors are equal and, in practice, the benefits of TP and TN outcomes may also differ. In a 2-class problem, one can construct a cost-benefit matrix that has diagonal elements representing the benefits of correct classification decisions (TP and TN) and off-diagonal elements corresponding to the costs of incorrect classification decisions (FP and FN), as shown in Table 1. In the case of insemination events for lactating dairy cows, TP corresponds to the correct decision to inseminate a cow that will conceive, $\mathrm{TN}$ corresponds to the correct decision to refrain from inseminating a cow that would not have conceived, FP corresponds to the incorrect decision to inseminate a cow that will not conceive, and FN corresponds to the incorrect decision to refrain from inseminating a cow that would have conceived.

The precise assignment of costs and benefits associated with TP, TN, FP, and FN decisions can be the subject of considerable debate, but it is clear that these values will be influenced by cow-level factors such as DIM and RMY, herd-level factors such as 21-d pregnancy rate and culling rate, and population-level parameters such as heifer price and salvage value. In the present study, we sought to demonstrate the concepts of lift chart analysis and cost-sensitive evaluation of classifiers using realistic economic values (see below) for commercial herds in Wisconsin, and we recognize that determination of the exact costs and benefits for a specific herd could easily justify another research study.

The cost-benefit matrix was constructed using the estimates of pregnancy values and cost of days open from a dynamic programming (DP) model developed by Kalantari and Cabrera (2012). In that study, DP was used to find optimal replacement decisions; however, to do so, one must determine the economic value of each individual cow. In our situation, these cow values can be used to calculate the value of a pregnancy and the cost of an additional day open. A brief explanation of the model follows:

Optimization in DP starts at the end of the planning horizon, by setting the values of the cows to their carcass values, and moves backward in monthly steps while calculating total expected discounted revenue $\left[f_{i}(n)\right]$ using a recursive formula (Kristensen, 1996):

$$
f_{i}(n)=\max _{d}\left\{r_{i}^{d}+\beta \sum_{j=1}^{u} p_{i j}^{d} f_{j}(n-1)\right\}, \quad i=1, \ldots, u,
$$

where $d$ is our decision (keep vs. replace), $i$ is the state variable (RMY, parity, month in milk, and month of pregnancy) describing the cows in the model, $\beta$ is the discount factor $[1 /(1+r)$, where $r$ is the monthly interest rate), $u$ is the total number of states, and $p_{i j}^{d}$ are transition probabilities among cow states for a given decision $(d)$. After reaching the current stage, the values that are obtained reflect the values of cows in the herd. These cow values can be used to calculate the pregnancy values, which represent the differences between individual cows that are pregnant or non-pregnant for a given set of state variables. A detailed description of the DP model can be found in Kalantari and Cabrera (2012).

As noted earlier, medium RMY corresponds to cows with milk yield between $6 \%$ below and $6 \%$ above the within-herd mean, low RMY corresponds to cows with milk yield between 18 and $6 \%$ below the within-herd mean, and high RMY refers to cows with milk yield between 6 and $18 \%$ above the within-herd mean. Figure 2 illustrates the conceptual trajectories during the lactation for the value of a nonpregnant cow (solid line) and the value of a pregnant cow (dashed line); actual values for these curves were calculated by Kalantari and Cabrera (2012) and used to derive the cost-benefit matrix in the following manner. For a TP classification, the benefit corresponds to the difference between 
the value of a pregnant cow at point $\mathrm{C}$ on the dashed curve (1 mo after the insemination, when diagnosed pregnant) and the value of a nonpregnant cow at point $\mathrm{B}$ on the solid curve $(1 \mathrm{mo}$ after the insemination, when diagnosed as nonpregnant). In the case of a $\mathrm{TN}$ classification, the benefit corresponds to the difference between the value of a nonpregnant cow at point $\mathrm{B}$ on the solid line (1 mo after the insemination, when diagnosed as nonpregnant) and the value of a nonpregnant cow at point $\mathrm{A}$ on the solid line (at the time the cow was passed over for insemination). Moving from point $\mathrm{A}$ in a given month to point $\mathrm{B}$ in the subsequent month is an inevitable outcome that is associated with a reduction in the value of a nonpregnant cow, but no misclassification occurred due to a poor prediction or an errant management intervention, so a benefit of $\$ 0$ was assumed.

Table 2 shows the pregnancy values derived from the DP model of Kalantari and Cabrera (2012) for each of the 9 DMI by RMY subclasses of Data_1, as well as pregnancy values for 150 to 180 DIM at each level of RMY, which contribute to costs assigned to FN classifications. A pregnancy value and loss of pregnancy cost of $\$ 88$ and $\$ 24.30$ were assumed for the cost-sensitive evaluation in Data_2, based on the average of values given in Table 2, respectively. As noted earlier, an insemination cost of $\$ 25$ (Olynk and Wolf, 2009) was used for both analyses.
Table 2. Pregnancy values (\$) assigned to primiparous Holstein cows in the Data_1 analysis, according to DIM and relative milk yield (RMY), as derived from the dynamic programming model of Kalantari and Cabrera $(2012)^{1}$

\begin{tabular}{lrcc}
\hline & \multicolumn{3}{c}{ RMY } \\
\cline { 2 - 4 } DIM & Low & Medium & High \\
\hline $60-90$ & 66 & 65 & 63 \\
$90-120$ & 89 & 87 & 86 \\
$120-150$ & 124 & 123 & 122 \\
$150-180$ & 149 & 148 & 147 \\
\hline
\end{tabular}

${ }^{1}$ Low RMY cows were between 6 and $18 \%$ below the within-herd mean; medium RMY cows were between $6 \%$ below and $6 \%$ above the withinherd mean; and high RMY cows were between 6 and $18 \%$ above the within-herd mean.

With regard to misclassified outcomes, the cost of a FP decision is depicted as the difference between point $\mathrm{B}$ on the solid curve for a nonpregnant cow (1 mo from now, when diagnosed as nonpregnant), and the point denoted as "-IC," which represents the insemination cost, which was incurred unnecessarily due to an incorrect model prediction. Conceptually, the most challenging case is the FN classification. In this case, the cow would have become pregnant if she had been inseminated, but she was not inseminated due to an incorrect model prediction. The opportunity cost for the incorrect decision can be calculated as the difference between points $\mathrm{C}$ and $\mathrm{D}$ on the dashed curve for

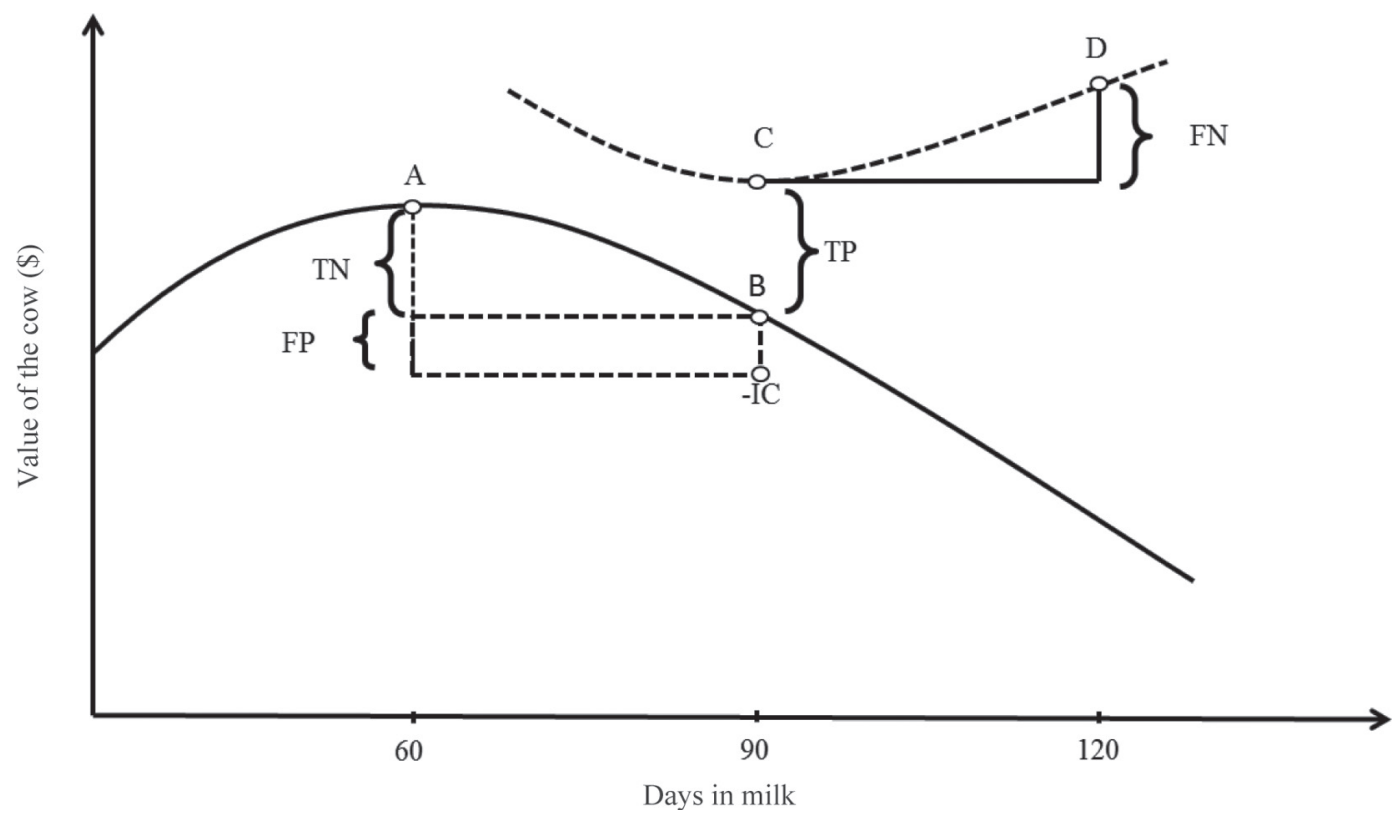

Figure 2. Conceptual curves that depict the value of a pregnant cow (dashed curve) and value of a nonpregnant cow (solid line) and value of a pregnant cow (dashed line), as used to assign the benefits of true positive (TP) and true negative (TN) classifications and costs of false positive (FP) and false negative (FN) classifications in the present study. Point A is the value of the cow at the time of insemination; points B and $\mathrm{C}$ are the values of nonpregnant and pregnant cows 1 mo after the insemination, respectively; and point $\mathrm{D}$ is the value of a pregnant cow 2 mo after the insemination, and IC is the insemination cost. 
a pregnant cow, where point $\mathrm{D}$ represents the value of a pregnancy 2 mo after the foregone insemination (the earliest possible time that the cow can be diagnosed pregnant if we postpone insemination for $1 \mathrm{mo}$ ). The assumption behind this is as follows; if we forego the opportunity to inseminate a cow that would have become pregnant, we will have another chance to inseminate that cow in the following month (assuming her probability of conception is similar and assuming that we make a correct decision). So, we can think of $\mathrm{FN}$ as the cost of deferring the pregnancy for $1 \mathrm{mo}$ or, in other words, the opportunity cost of delaying pregnancy for 1 mo. Readers should be careful not to confuse "pregnancy rate" and "outcome of insemination" because pregnancy rate is a herd-level statistic, whereas outcome of insemination is a cow-level statistic, which is the focus of our study - getting pregnant or not as the outcome of insemination is a Bernoulli (yes/no) variable. Therefore, if all other variables pertaining to this cow remain the same in the following month or cycle, the chance of that specific cow getting pregnant or not would remain constant, and the outcome will be either 0 or 1 . Also, note that in mid lactation, the values of TP and FN will typically be similar because the cow is still milking at a high level and, for example, the value of achieving pregnancy at $90 \mathrm{~d}$ is not much greater than the value of achieving pregnancy at 60 d. However, as the cow proceeds through the lactation and her production declines, the cost of deferring her pregnancy for 1 mo may be greater. In reality, the insemination in the following month may or may not be successful, and the probability of conception at the subsequent insemination may differ if the cow's health status or other explanatory variables change from one month to the next.

\section{Integration of Costs and Benefits into Classification Decisions}

Given a set of explanatory variables, a typical classification algorithm will assign a positive or negative outcome to a given insemination event based on an underlying probability threshold. In other words, if the probability of conception exceeds a certain value, the event will be classified as a success, and if it falls short of that value, it will be classified as a failure. A decision threshold of 0.5 is typically used to classify instances as success or failures. Alternatively, this threshold, which is known as the score threshold in the machine learning literature, can be optimized for a given set of cost values for incorrect decisions and benefit values for correct decisions. If a cost-benefit matrix with information about the values of TP, TN, FP, and FN classifications is available, this information can be coupled with the probability threshold to compute the costs associated with a particular classifier and probability threshold. More importantly, the optimum probability threshold at which benefits are maximized (or costs are minimized) can be determined. For example, if the decision in question is whether or not to inseminate a given cow, a cost-sensitive evaluation of the classifier can tell us the optimum number (and identities) of high-fertility cows that should be inseminated, as well as the corresponding number (and identities) of low-fertility cows that should not be inseminated, to maximize profitability of the reproductive management program. Or, more generally, such an evaluation could help us identify a subset of cows that should be inseminated with sexenhanced semen, a subset that should be inseminated with conventional semen, and a subset that should not be inseminated. In the present study, benefits and costs associated with TP, TN, FP, and FN classifications were taken into account when evaluating new insemination decisions, but not when training the classifier; this is what differentiates cost-sensitive evaluation from cost-sensitive learning (Witten and Frank, 2005). This study focused on cost-sensitive evaluation, because previous studies (Elkan, 2001) have shown that most of the gain associated with incorporating a cost-benefit matrix comes in the evaluation phase, rather than the learning phase (by cost-sensitive learning procedures, such as changing the balance of positive and negative training examples). Elkan (2001) recommends a 2-step process of training a classifier without considering cost and benefit information, followed by computing optimal decisions using probability estimates given by the classifier based on the cost-benefit matrix.

By applying the elements of the cost-benefit matrix to different probability thresholds along the ROC curve, the costs and benefits of each potential decision were evaluated. In Data_1, 10-fold cross-validation was used in conjunction with the cost-benefit matrix to determine the optimum probability threshold for each of the 9 DIM by RMY subclasses, assuming a strategy of maximizing total profit of the reproductive program. Estimates of the lift factor, as well as estimates of financial gains or losses, were subsequently evaluated in each of the 9 subclasses. In Data_2, the optimal probability threshold was also determined by 10 -fold crossvalidation, and model results were saved and applied to the aforementioned partition of 3,197 independent insemination events for estimation of financial gains and losses, to mimic a real application of this decisionmaking tool on a commercial farm. 


\section{RESULTS AND DISCUSSION}

Results of the Data_1 analysis, including classification accuracy, lift chart analysis, and cost-sensitive evaluation, are shown in Table 3 for each of the 9 DIM by RMY subclasses. As noted earlier, results are from 10-fold cross-validation within the full population of eligible cows in each of the 9 subclasses. When the benefits and costs associated with various types of correctly classified and misclassified events were not considered, the accuracy of classifying upcoming insemination events as successes or failures (i.e., the proportion of correctly classified events) ranged from 0.58 to 0.80 , whereas area under the ROC curve ranged from 0.61 to 0.80 . No clear trends were observed between classification accuracy and DIM or RMY.

In the subsequent lift chart analysis of Data_1, which is also shown in Table 3, conception rates in selected subpopulations of highly fertile cows within each DIM by RMY subclass ranged from 0.35 to 0.51 . These values were consistently greater than conception rates in the respective populations of all eligible cows, which ranged from 0.32 to 0.44 . The corresponding lift factors ranged from 1.02 to 1.15 , which suggests that financial gains might be achieved by focusing semen, labor, and supplies (e.g., for timed AI) on a selected subset of highly fertile cows rather than the entire population of eligible cows.

Table 3 also shows results of the cost-sensitive evaluation of correctly classified and misclassified insemination events for each of the 9 DIM by RMY subclasses, which was implemented using the cost-benefit matrix data shown in Table 2. When costs and benefits associated with classification decisions were considered, the accuracies of prediction achieved by 10 -fold cross-validation ranged from 0.42 to 0.57 . These accuracies tended to be lower than those achieved when costs and benefits were ignored, because in this case the algorithm chooses a score threshold that maximizes the profit derived from correct and incorrect decisions, rather than the score threshold, which simply maximizes prediction accuracy. The optimum probability threshold (also known as the score threshold) reflects the point at which total profit of the reproductive management program is maximized given a particular cost-benefit matrix and considering the algorithm's ability to identify various high-fertility subsets of cows in the lift chart analysis. The selected subsets of highly fertile cows represented from 79.0 to $97.2 \%$ of the eligible cows in the population, depending on the DIM by RMY subclass. This means that breeding the vast majority of cows was typically justified, presumably because insemination costs were low relative to the value of a pregnancy, noting that low insemination costs reflect the fact that semen of AI bulls used on commercial dairy farms in the United States tends to be inexpensive. Higher insemination costs (e.g., due to the use of sex-enhanced semen) would likely result in selection of a smaller percentage of cows for insemination. The target population refers to the group of cows that would conceive if they were inseminated, and this would be composed of TP and FN classifications. In our cost-sensitive evaluation, 90.3 to $99.0 \%$ of cows in the target population were identified as candidates to be inseminated by the classification algorithm, so very few cows were missed as FN classifications. The apparent contradiction between the identification of a very high percentage of the target population while achieving relatively modest accuracy of prediction reflects the large number of FP classifications. Such classifications occur frequently due to low insemination costs coupled with high pregnancy values, as noted earlier, which mean that FP classifications tend to be inexpensive mistakes. Profit was calculated for the optimal reproductive management strategy, as determined by the lift analysis and cost-sensitive evaluation process, as well as for the base strategy, in which all eligible cows were inseminated. Gains in profit were achieved in all 9 DIM by RMY subclasses by focusing inseminations on the subset of high-fertility cows, as identified by the algorithm described herein. These gains ranged from $\$ 2,783$ for the population of 2,291 eligible high-RMY cows at 120 to 150 DIM, to $\$ 15,123$ for the population of 13,745 eligible low-RMY cows at 60 to 90 DIM in a given breeding period (1 mo in our case, though one could do such an analysis in 21-d increments if desired). When expressed as the gain in profit per eligible cow, the gains ranged from a low of $\$ 0.44$ per eligible medium-RMY cow at 60 to 90 DIM, to a high of $\$ 2.18$ per eligible high-RMY cow at 90 to 120 DIM in a given breeding period. As noted earlier, the actual gain that could be achieved on a particular farm will depend on the cost-benefit matrix associated with that farm, which will be a function of pregnancy rate, culling rate, replacement heifer inventory, and related factors. The gain will also depend on the algorithm's ability to accurately differentiate successes and failures before the insemination, given a set of explanatory variables, and this will depend critically on the accuracy and completeness of data recording for these explanatory variables.

Because the accuracy and completeness of data recording differs widely between farms, and because routine implementation of the decision-support tool described herein will likely involve centralized model development and parameter tuning with subsequent application by field consultants, we carried out a second analysis using Data_2. That analysis, for which the results are given in Table 4, involved development and 
Table 3. Classification accuracy and results of the lift chart analysis and cost-sensitive evaluation in Data_1, according to DIM and relative milk yield (RMY), when maximizing total profit of the reproductive management program

\begin{tabular}{|c|c|c|c|c|c|c|c|c|c|}
\hline \multirow[b]{2}{*}{ Item } & \multicolumn{3}{|c|}{60 to $90 \mathrm{DIM}$} & \multicolumn{3}{|c|}{90 to $120 \mathrm{DIM}$} & \multicolumn{3}{|c|}{120 to 150 DIM } \\
\hline & $\begin{array}{l}\text { Low } \\
\text { RMY }\end{array}$ & $\begin{array}{l}\text { Medium } \\
\text { RMY }\end{array}$ & $\begin{array}{l}\text { High } \\
\text { RMY }\end{array}$ & $\begin{array}{l}\text { Low } \\
\text { RMY }\end{array}$ & $\begin{array}{l}\text { Medium } \\
\text { RMY }\end{array}$ & $\begin{array}{l}\text { High } \\
\text { RMY }\end{array}$ & $\begin{array}{l}\text { Low } \\
\text { RMY }\end{array}$ & $\begin{array}{l}\text { Medium } \\
\text { RMY }\end{array}$ & $\begin{array}{l}\text { High } \\
\text { RMY }\end{array}$ \\
\hline \multicolumn{10}{|l|}{ Classification accuracy } \\
\hline Eligible population (n) & 13,745 & 11,015 & 5,239 & 6,109 & 5,271 & 2,372 & 4,576 & 4,188 & 2,291 \\
\hline Accuracy of prediction & 0.68 & 0.58 & 0.72 & 0.74 & 0.72 & 0.74 & 0.75 & 0.76 & 0.80 \\
\hline Area under the $\mathrm{ROC}^{1}$ curve & 0.75 & 0.61 & 0.80 & 0.78 & 0.77 & 0.77 & 0.76 & 0.78 & 0.80 \\
\hline \multicolumn{10}{|l|}{ Lift chart analysis } \\
\hline $\mathrm{CR}^{2}$ in the eligible population & 0.44 & 0.44 & 0.44 & 0.37 & 0.36 & 0.34 & 0.33 & 0.32 & 0.34 \\
\hline $\mathrm{CR}$ in the selected subpopulation & 0.49 & 0.45 & 0.51 & 0.43 & 0.41 & 0.41 & 0.36 & 0.36 & 0.35 \\
\hline Lift factor & 1.11 & 1.02 & 1.15 & 1.15 & 1.13 & 1.20 & 1.09 & 1.13 & 1.04 \\
\hline \multicolumn{10}{|l|}{ Cost-sensitive evaluation } \\
\hline Accuracy of prediction & 0.56 & 0.46 & 0.57 & 0.50 & 0.49 & 0.52 & 0.42 & 0.45 & 0.46 \\
\hline Optimal probability threshold & 0.11 & 0.10 & 0.14 & 0.10 & 0.09 & 0.10 & 0.05 & 0.06 & 0.05 \\
\hline$\%$ of eligible population & 85.5 & 97.2 & 84.5 & 83.6 & 85.3 & 79.0 & 89.9 & 85.6 & 87.0 \\
\hline$\%$ of target population & 95.7 & 99.0 & 97.2 & 96.1 & 96.7 & 95.0 & 97.8 & 96.9 & 90.3 \\
\hline Profit with optimal strategy $(\$)^{3}$ & 260,334 & 196,495 & 96,841 & 130,413 & 105,504 & 43,210 & 130,963 & 114,116 & 67,573 \\
\hline Profit with inseminating all cows $(\$)^{3}$ & 245,211 & 191,661 & 86,548 & 124,196 & 97,619 & 38,047 & 125,932 & 107,883 & 64,790 \\
\hline Total gain in profit $(\$)^{3}$ & 15,123 & 4,834 & 10,293 & 6,217 & 7,885 & 5,163 & 5,031 & 6,233 & 2,783 \\
\hline Gain in profit per eligible cow $(\$)^{3}$ & 1.10 & 0.44 & 1.96 & 1.19 & 1.50 & 2.18 & 1.10 & 1.49 & 1.21 \\
\hline
\end{tabular}

Receiver operating characteristic.

${ }^{2}$ Conception rate.

${ }^{3}$ In a given breeding period (1 mo). 
Table 4. Results of cost-sensitive evaluation and lift chart analysis for 3,197 independent insemination events in Data_2, with the objective of maximizing total profit of the reproductive management program

\begin{tabular}{lc}
\hline Item & Value \\
\hline Classification accuracy & 14,000 \\
Eligible population for training (no.) & 0.80 \\
Accuracy of prediction & 0.89 \\
Area under the ROC ${ }^{1}$ curve & \\
& \\
Lift chart analysis & 0.43 \\
CR ${ }^{2}$ in the eligible population & 0.66 \\
CR in the selected subpopulation & 1.55 \\
Lift factor & \\
& 3,197 \\
Cost-sensitive evaluation & 0.76 \\
Eligible population for validation $($ no. $)$ & 0.33 \\
Accuracy of prediction & 0.59 \\
Optimal probability threshold & 0.92 \\
$\%$ of eligible population & 91,354 \\
Prof target population with optimal strategy $(\$)^{3}$ & 74,694 \\
Profit with inseminating all cows $(\$)^{3}$ & 16,660 \\
Total gain in profit $(\$)^{3}$ & 5.21 \\
Gain in profit per eligible cow $(\$)^{3}$ & \\
\hline
\end{tabular}

${ }^{1}$ Receiver operating characteristic.

${ }^{2}$ Conception rate.

${ }^{3} \mathrm{In}$ a given breeding period $(1 \mathrm{mo})$.

tuning of the prediction model in a randomly selected partition of 14,000 insemination events on 3 farms with high quality data regarding infectious diseases, such as mastitis, and early postpartum metabolic disorders, such as ketosis. Very high accuracy of prediction was observed in these herds, with proportion of correctly classified insemination events of 0.80 and area under the ROC curve of 0.89 . Note that accuracies and areas under the ROC curve ranged from 0.58 to 0.80 and from 0.61 to 0.80 , respectively, in Data_1.

Greater accuracy of prediction should lead to a more informative lift chart, and Table 4 shows the lift chart analysis and cost-sensitive evaluation results corresponding to implementation of the aforementioned model in the 3,197 randomly chosen independent insemination events that comprised the validation population. One can see that conception rate in the selected subpopulation of highly fertile cows was 0.66 compared with 0.43 in the entire population of eligible cows, which results in a remarkably high lift factor of 1.55. When the costs and benefits of misclassified and correctly classified insemination events were taken into account, using a pregnancy value averaged across DIM and RMY subclasses in Table 2, the accuracy of prediction was 0.76 for an optimum probability (or score) threshold of 0.33 . In this application, only $59 \%$ of the eligible population of cows was inseminated, yet these cows represented $92 \%$ of the target population of cows that would have conceived if inseminated. In terms of profit, the total gain achieved by using the optimized reproductive management strategy derived herein, relative to the alternative of breeding all 3,197 eligible cows, was $\$ 16,660$ in a given breeding period. On a per cow basis, the gain in profit was $\$ 5.21$ per eligible cow in a given breeding period, which was several-fold greater than the gains achieved with lower quality data, and hence poorer accuracy of prediction, in Data_1. This difference demonstrates the importance of accuracy and completeness of recording of potential explanatory variables, with respect to devising more profitable reproductive management programs.

Although it is true that data on many farms are incomplete or inaccurate, especially as regards health events, a substantial number of modern dairy farms have strictly enforced standard operating procedures, meticulous recording of data, and new technologies for automated capture of health, management, and performance information. Thus, although the results for Data_2 reflected the benefit of more accurate and complete health history data for individual cows compared with herds in Data_1, there is still significant room for improvement in herds such as those represented in Data_2. For example, although the present study considered the genetic predisposition of individual cows using PTA for daughter pregnancy rate, further gains could be achieved by using genomic predictions if most or all eligible cows were genotyped (Weigel et al., 2012). Similarly, whereas the present study considered each cow's health history as recorded in the on-farm herd management software, additional gains could be achieved by using health-related indicators captured by physical activity and rumination monitoring systems. And finally, although the present study considered variables such as milk yield and fat:protein ratio at the most recent DHI test, future applications of this methodology might utilize milk weights, electrical conductivity, or mid-infrared spectral data recorded on a daily basis.

Last, as noted earlier, strategies for maximizing the profit of reproductive management programs may differ widely between farms, depending on current performance, financial position, or future plans. For example, a herd that is expanding may face a shortage of replacement heifers, which could change the structure of the cost-benefit matrix for that herd. Conversely, if replacement heifers are plentiful and inexpensive, this could lead to a much different cost-benefit matrix and, in turn, a different optimization strategy. In theory, a $3 \times 3$ (or larger) cost-benefit matrix could be implemented; for example, to classify eligible cows or heifers into subclasses corresponding to sex-enhanced semen, conventional semen, or do-not-breed (or use beef semen) reproductive management actions at a given time. However, this would increase the complexity, par- 
ticularly as regards determination of appropriate cost and benefit parameters, which in this case would lead to a $3 \times 3$ or a larger cost-benefit matrix to represent all correct and incorrect classifications. Fruitful topics for future research projects could include an assessment of the extent of variation in cost and benefit parameters between herds, as well as variation in these parameters within a herd over time, in addition to an evaluation of the sensitivity of recommended reproductive management actions to errors in the cost-benefit matrix. Considering retention pay-off of each cow (Shahinfar et al., 2014a) as an important economic measurements of cow productivity at the time of decision making, to inseminate a cow or not can further optimize the profitability of the farm more precisely. Previous authors have recommended a delay in inseminating high-producing primiparous cows, because the pregnancy value associated with these highly persistent and fertile cows tends to be negative in early lactation (DeVries, 2006); however, Inchaisri et al. (2011) showed that extending the voluntary waiting period to more than $6 \mathrm{wk}$ is associated with economic losses on Dutch dairy farms, which is in line with the results of Steeneveld and Hogeveen (2012), who that found a small economic effect of delaying insemination. Furthermore, McCullock et al. (2013) showed that profitability is enhanced by using sex-enhanced semen on yearling heifers and a fraction of genetically superior cows, and Seidel (2003) noted that sex-enhanced semen could be used to improve the genetic merit of herd replacements when its usage was targeted toward genetically superior cows or heifers. Although the present study did not consider genetic merit of the cows when constructing the cost-benefit matrix, optimizing costs and benefits of insemination decisions involving cows of high or low genetic merit in the presence of sex-enhanced semen could be the subject of a future study. When semen is inexpensive and technician costs are modest, the common practice is to simply inseminate all eligible cows and ignore the costs associated with breeding lowly fertile cows with a history of health and reproductive problems. Implementation of such a decision-making tool would likely require the assistance of a professional reproductive management consultant. The rapid development and adoption of systems and devices for real-time monitoring of animal health, activity, and performance will enhance our ability to refine, and derive greater benefit from, the types of decision-support tools described herein.

\section{Sensitivity Analysis}

The effect of changes $( \pm 20 \%)$ in components of costbenefit matrix on the profit, total economic gain, and gain per eligible cow is shown in Table 5. Increasing the value of TP (pregnancy value) by $20 \%$ increased profit by $24 \%$ and increased total gain and gain per eligible cow by $132 \%$. On the other hand, a $20 \%$ decrease in the value of TP led to reductions in profit of similar magnitude. Changing the base level of FP cost by $\pm 20 \%$ caused a $0.6 \%$ change in total profit and $3.3 \%$ change in gain in profit, whereas changing FN cost by $\pm 20 \%$ changed the total profit by $3.5 \%$ and gain in profit by $19 \%$. Thus, variation in pregnancy value (TP) has the largest effect on predictions, whereas variation in insemination cost (FP) has a relatively small effect on profitability. In practice, the values of TP, TN, FP, and FN classifications may vary by farm or over time. For example, herds that practice rotational grazing and seasonal calving might have significantly greater benefits for TP classifications and substantially greater costs for FN classifications.

\section{CONCLUSIONS}

Although prediction of the outcome of an insemination event for a lactating dairy cow from dozens of potential explanatory variables is difficult, information regarding genetic predisposition, health history, lactation performance, and other factors can be used with modern analytical tools, such as machine learning algorithms, to identify cows with substantially higher or lower probability of conception compared with the

Table 5. Sensitivity analysis applied to Data_2, with changes total profit, gain in profit per breeding period, and gain in profit per eligible cow per breeding period when varying elements of the cost-benefit matrix by $\pm 20 \%$

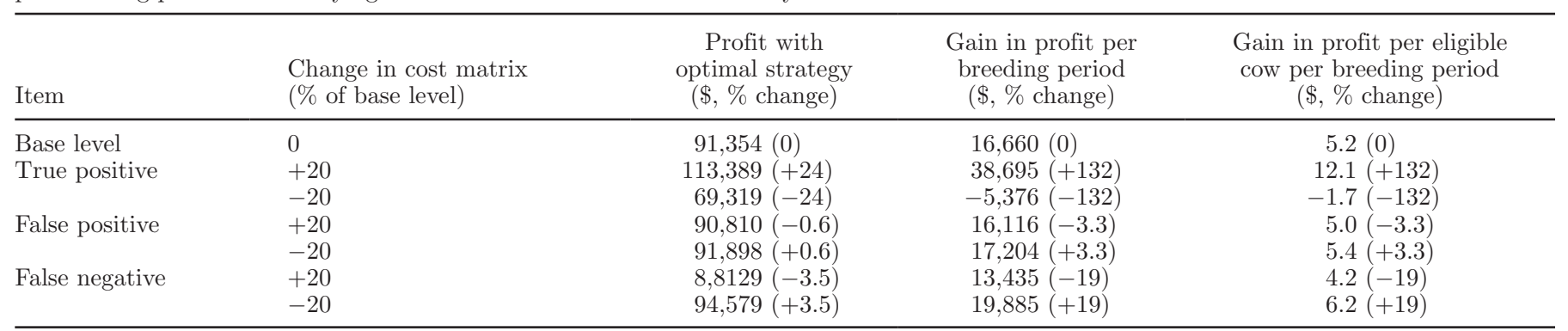


average of the population of eligible cows. This research showed that quality of data, particularly health traits, can have a significant effect on accuracy of prediction of outcome of insemination in dairy cows. Lift chart analysis can be used to stratify the pool of eligible cows into those that are likely to conceive and those that should not be inseminated at a given point in time. When coupled with cost-sensitive evaluation of misclassified and correctly classified insemination events, the approach provides a potentially powerful tool to optimize the reproductive management strategy of a given farm under that farm's economic conditions. When high quality data were available, accuracy of 0.80 and area under the ROC curve of 0.89 were achieved, as well as a conception rate of 0.66 in the selected subpopulation of highly fertile cows. The lift chart analysis identified $59 \%$ of eligible cows, which represented $92 \%$ of the target population, and this provided a gain of $\$ 5.21$ per eligible cow in each (monthly) breeding period. The key features of this approach separately or combined with a retention pay-off calculation could be incorporated into existing dairy herd management software programs in the future.

\section{ACKNOWLEDGMENTS}

This project was supported by Agriculture and Food Research Initiative Competitive Grant no. 2010-8512220612 from the USDA National Institute of Food and Agriculture (Washington, DC). Data for this study were provided by Alta Genetics (Watertown, WI) and the USDA-ARS Animal Improvement Programs Laboratory (Beltsville, MD). Authors acknowledge the technical comments and support from Jon Schefers (Department of Dairy Science, University of Wisconsin-Madison) and David Vanness (Department of Population Health Sciences, University of Wisconsin-Madison). Kent Weigel acknowledges partial financial support from the National Association of Animal Breeders (Columbia, $\mathrm{MO})$.

\section{REFERENCES}

Britt, J. H. 1985. Enhanced reproduction and its economic implications. J. Dairy Sci. 68:1585-1592.

Chen, W., C. Hsu, and J. Hsu. 2011. Optimal selection of potential customer range through the union sequential pattern by using a response model. Expert Syst. Appl. 38:7451-7461.

De Vries, A. 2006. Economic value of pregnancy in dairy cattle. J. Dairy Sci. 89:3876-3885.

Elkan, C. 2001. The foundations of cost-sensitive learning. Pages 973978 in Proc. 17th Int. Joint Conf. Artificial Intelligence. Morgan Kaufmann, Seattle, WA.
Garverick, H. A., M. N. Harris, R. Vogel-Bluel, J. D. Sampson, J. Bader, W. R. Lamberson, J. N. Spain, M. C. Lucy, and R. S. Youngquist. 2013. Concentrations of nonesterified fatty acids and glucose in blood of periparturient dairy cows are indicative of pregnancy success at first insemination. J. Dairy Sci. 96:181-188.

Giordano, J. O., P. M. Fricke, M. C. Wiltbank, and V. E. Cabrera 2011. An economic decision-making support system for selection of reproductive management programs on dairy farms. J. Dairy Sci. 94:6216-6232.

Giordano, J. O., A. S. Kalantari, P. M. Fricke, M. C. Wiltbank, and V. E. Cabrera. 2012. A daily herd Markov-chain model to study the reproductive and economic impact of reproductive programs combining timed artificial insemination and estrus detection. J. Dairy Sci. 95:5442-5460.

Inchaisri, C., R. Jorritsma, P. L. A. M. Vos, G. C. van der Weijden, and H. Hogeveen. 2011. Analysis of the economically optimal voluntary waiting period for first insemination. J. Dairy Sci. 94:3811-3823.

Kalantari, A. S., and V. E. Cabrera. 2012. The effect of reproductive performance on the dairy cattle herd value assessed by integrating a daily dynamic programming model with a daily Markov chain model. J. Dairy Sci. 95:6160-6170.

Kristensen, A. R. 1996. Textbook notes of herd management: dynamic programming and Markov decision processes. Dina Notat No. 49, Department of Animal Science and Animal Health, Royal Veterinary and Agricultural University, Copenhagen, Denmark.

Lima, F. S., A. De Vries, C. A. Risco, J. E. P. Santos, and W. W. Thatcher. 2010. Economic comparison of natural service and timed artificial insemination breeding programs in dairy cattle. J. Dairy Sci. 93:4404-4413.

Ludwick, T. M., and E. R. Rader. 1968. Diagnosis of early pregnancy in cattle by ovarian analysis. J. Dairy Sci. 51:74-77.

McCullock, K., D. L. K. Hoag, J. Parsons, M. Lacy, G. E. Seidel Jr., and W. Wailes. 2013. Factors affecting the economics of using sexed semen in dairy cattle. J. Dairy Sci. 96:6366-6377.

Meadows, C., P. J. Rajala-Schultz, and G. S. Frazer. 2005. A spreadsheet-based model demonstrating the nonuniform economic effects of varying reproductive performance in Ohio dairy herds. J. Dairy Sci. 88:1244-1254.

Mitchell, T. M. 1997. Machine Learning. McGraw-Hill, New York, NY.

Olynk, N. J., and C. A. Wolf. 2009. Stochastic economic analysis of dairy cattle artificial insemination reproductive management programs. J. Dairy Sci. 92:1290-1299.

Seidel, G. E. Jr. 2003. Economics of selecting for sex: The most important genetic trait. Theriogenology 59:585-598.

Shahinfar, S., A. S. Kalantari, V. Cabrera, and K. Weigel. 2014a. Short communication: Prediction of retention pay-off using a machine learning algorithm. J. Dairy Sci. 97:2949-2952.

Shahinfar, S., C. D. Page Jr., J. N. Guenther, V. E. Cabrera, P. M. Fricke, and K. A. Weigel. 2014b. Prediction of insemination outcomes in Holstein dairy cattle using alternative machine learning algorithms. J. Dairy Sci. 97:731-742.

Sharma, A. K., C. J. Wilcox, F. G. Martin, and W. W. Thatcher 1990. Effects of stage of lactation and pregnancy and their interactions on milk yield and constituents. J. Dairy Sci. 73:1586-1592.

Sheng, V. S., B. Gu, W. Fang, and J. Wu. 2014. Cost-sensitive learning for defect escalation. Knowl. Base. Syst. 66:146-155.

Shin, H., and S. Cho. 2006. Response modeling with support vector machine. Expert Syst. Appl. 30:746-760.

Steeneveld, W., and H. Hogeveen. 2012. Economic consequence of immediate or delayed insemination of a cow in oestrous. Vet. Rec. 171:17. http://dx.doi.org/10.1136/vr.100183.

Weigel, K. A., P. C. Hoffman, W. Herring, and T. J. Lawlor. 2012. Potential gains in lifetime net merit from genomic testing of cows, heifers, and calves on commercial dairy farms. J. Dairy Sci. 95:2215-2225.

Witten, I. H., and E. Frank. 2005. Evaluating what's been learned. Pages 164-165 in Data Mining. 2nd ed. Elsevier, San Francisco, CA. 